The Be Phenomenon in Early-Type Stars, IAU Colloquium 175

ASP Conference Series, Vol. 214, 2000

M. A. Smith, H. F. Henrichs, and J. Fabregat, eds.

\title{
B-type Emission-line Stars with Warm Circumstellar Dust
}

\author{
T.A. Sheikina \\ Fesenkov Astrophysical Institute, Kamenskoe plato, 480068, Almaty, \\ Kazakhstan
}

A.S. Miroshnichenko

Ritter Observatory, University of Toledo, Dept. of Physics and Astronomy, Toledo, $\mathrm{OH} 43606$, USA

\section{P. Corporon}

Département de Physique, Université of Montréal, C.P. 6128, Succ. A, Montréal, HзC ЗJ\%, Canada

\begin{abstract}
We selected a group of $11 \mathrm{~B}$-type emission-line stars with large IR excesses, which show a steep flux decrease towards longer wavelengths. Analysis of their observational characteristics has been carried out on the basis of the data collected from literature, results of our recent photometric and high-resolution spectroscopic observations. Possible nature and evolutionary state of the group objects are discussed.
\end{abstract}

Among the IRAS sources associated with early-type emission-line stars there is a small group with a steep decrease of the IR flux longward of $25 \mathrm{mi}$ crons $([25]-[12] \leq 0.1,[60]-[25] \leq-0.3)$. Such IRAS colors usually have late-type stars surrounded by a small amount of circumstellar dust. Most of the selected objects (e.g. HD 45677, HD 50138, MWC 84, MWC 342) have been classified as $\mathrm{B}[\mathrm{e}]$ stars with uncertain evolutionary state. Their main features are lack of photospheric absorption and forbidden emission lines; very strong Balmer line emission; strong IR excesses, which imply the presence of circumstellar dust not cooler than $\sim 200 \mathrm{~K}$; mass loss rates of the order of $10^{-6} \mathrm{M}_{\odot} \mathrm{yr}^{-1}$. The objects form two distinct groups in the HR diagram (Bergner et al. 1990, Miroshnichenko et al. 1997, Zorec 1998, de Freitas Pacheco 1998). CPD-57²874, CPD $-52^{\circ} 9243$, HDE 327083 , MWC 300 are located close to the HumphreysDavidson limit, while the other stars near the zero-age main-sequence (see Table 1).

We completed the group with several recently selected stars, collected the data available in the literature, and obtained new observations in order to get a comprehensive view on these puzzling stars. The questions we address in this study are as follows. Why the dust around these objects occupies a compact region, while other massive stars (e.g., Herbig Ae/Be stars, LBVs) display much more extended dusty envelopes? What is the origin of the enhanced mass loss? 


\section{Observations}

Observations of these unusual objects are still rare. During 1993-1999 we obtained the following observational data: simultaneous Johnson $U B V R I J H K$ photometry at the 1-meter telescope of the Tien-Shan Observatory with a twochannel photometer-polarimeter of the Pulkovo Observatory (Bergner et al. 1988); quasi-simultaneous Cousins $U B V R I$ and $J H K L$ photometry at the 0.5and 0.75-meter telescopes of the SAAO; high-resolution spectroscopy (3900-6800 $\AA, R \sim 38000$ ) at the 1.93-meter telescope of the Observatoire de Haute Provence with a spectrograph ÉLODIE (Corporon \& Lagrange 1999).

Table 1. Parameters of the sources

\begin{tabular}{rrlclcccr}
\hline Name & $V$ & Sp.T. & D & $\log \mathrm{L}_{*}$ & $\mathrm{E}_{B-V}^{t o t}$ & 5780 & $\mathrm{E}_{B-V}^{I S}$ & $\mathrm{H} \alpha$ \\
\hline AS 78 & 11.3 & B2-4 & 2.5 & $3.9 \pm 0.1$ & 0.90 & 0.43 & 0.8 & 115 \\
CI Cam & 11.6 & O9-B0 & 1.5 & $3.5 \pm 0.5$ & 1.10 & 0.29 & 0.5 & $250^{\mathrm{a}}$ \\
HD 45677 & 8.0 & B2 & 0.5 & $3.5 \pm 0.4$ & 0.20 & 0.02 & 0.04 & 170 \\
HD 50138 & 6.6 & B5-8 & 0.3 & $2.9 \pm 0.2$ & 0.15 & & & 60 \\
$-57^{\circ} 2874$ & 10.1 & B3-5 & 2.5 & 5.7 & 1.85 & & & \\
$-52^{\circ} 9243$ & 10.3 & B3-4 & 4.9 & $5.7 \pm 0.3$ & 1.75 & 0.77 & 1.4 & $57^{\mathrm{b}}, 83^{\mathrm{c}}$ \\
HDE 327083 & 9.7 & B3-6 & 5.0 & 6.3 & 1.80 & & & 36 \\
MWC 300 & 11.6 & B1 & 15 & 5.7 & 1.20 & 0.40 & 0.8 & $140 \pm 10$ \\
MWC 623 & 10.6 & B2 & 2.4 & 4.1 & 1.40 & 0.75 & 1.4 & $122 \pm 5^{\text {d }}$ \\
MWC 342 & 10.5 & B0-1 & 1.0 & $4.1 \pm 0.4$ & 1.40 & 0.29 & 0.5 & $200 \pm 15$ \\
MWC 657 & 12.5 & B0-1 & 2.0 & $3.7 \pm 0.3$ & 1.60 & 0.54 & 1.0 & 180 \\
\hline
\end{tabular}

$D$ is the distance from the Sun in kpc (column 4), bolometric luminosity is given in solar units (column 5). $\mathrm{E}_{B-V}^{\text {tot }}$ is the overall reddening determined from the observed and intrinsic color-indices, $\mathrm{E}_{B-V}^{I S}$ is the interstellar reddening derived from the equivalent width of the $5780 \AA$ interstellar band. The equivalent widths of the $5780 \AA$ band (column 7) and $\mathrm{H} \alpha$ (column 9) are given in $\AA$. ${ }^{\text {a }}$ - the value measured during the quiescence state, ${ }^{b}$ from Lopes et al. (1992), c - from Winkler \& Wolf (1989), ${ }^{\mathrm{d}}-$ Zickgraf \& Stahl (1989) give $\mathrm{EW}(\mathrm{H} \alpha)=170 \AA$.

\section{Analysis of the observational data}

Most of the objects are very reddened. MWC 342, MWC 623 and MWC 657 lie above the interstellar reddening vector for the hottest stars, i.e. contribution of both free-free and free-bound radiation is large. In the IRAS color-color diagram all the objects are located in the region occupied mainly by cool stars. Very weak or absent $10 \mu \mathrm{m}$ silicate emission features in their spectra point to either the presence of C-rich circumstellar dust or a very high optical depth. In the latter case, the dust should have a disk-like distribution, since we see the stars. The Balmer line profiles of all the objects except CPD $-52^{\circ} 9243$ and AS 
78 do not show components below the continuum level, i.e. circumstellar gas distribution is also essentially non-spherical. Different strengths of the central absorption may be due to different inclination angles of the disks to the line of sight. IRAS images processed with a $15^{\prime \prime}$ pixel $^{-1}$ resolution are point-like for all the sources. Most of them are barely seen or absent already at $60 \mu \mathrm{m}$ that indicates the absence of cold dust.

The interstellar contribution to the overall reddening was estimated from the strength of the diffuse interstellar bands (DIB). A good relationship exists between the equivalent width of the DIB at $5780 \AA$ and color-excess $\mathrm{E}_{B-V}^{I S}$ (Herbig 1993). This method does not give precise results but it indicates if the circumstellar reddening is present or not. A large discrepancy between the overall and interstellar color-excesses is found for MWC $300, \mathrm{CPD}-52^{\circ} 9243, \mathrm{CI}$ Cam, MWC 342, and MWC 657.

\section{Discussion}

The binarity of MWC 623 (B2V+K2II-III, Zickgraf \& Stahl 1989), recent X-ray outburst of CI Cam (Frontera et al. 1998), and positional closeness of an Xray source to MWC 342 (Miroshnichenko 1991) suggest that the low-luminosity objects may be binary stars. Such a scenario may also be invoked to explain the existence of a disk around B[e] supergiants (Langer \& Heger 1998). However, the presence of the secondary components (except for MWC 623) is seen neither in the visible spectra (e.g. Corporon \& Lagrange 1999) nor in the photometric behavior (de Winter \& van den Ancker 1997). Thus, the inferred companion star should be much fainter than the massive primary.

The low-luminosity objects might be similar to $\mathrm{Be} / \mathrm{X}$-ray binaries. However, primary components of the latter being surrounded by dense equatorial disks (e.g. Telting et al. 1998) do not exhibit radiation of circumstellar dust, therefore their disks are too compact to have outer temperatures permitting dust formation $(\sim 1500 \mathrm{~K})$. Hence, the binary separation should be larger in the objects of our sample. The IR spectral energy distributions longward of 25 $\mu \mathrm{m}$ are consistent with those of optically thick disks with outer temperatures of 200 K (Lynden-Bell \& Pringle 1974).

Three of the four supergiant candidates (CPD-52 $9243, \mathrm{CPD}-57^{\circ} 2874$, and HDE 327083) show an extremely high reddening, most of which is interstellar. They are most likely distant and, hence, luminous objects. ISO spectra of HDE 327083 and CPD-57 2874 contain mainly amorphous structures, which are expected in evolved stars (Voors 1999). The fourth object, MWC 300, still have a controversial status. Wolf \& Stahl (1985) estimated its $M_{V} \leq-8^{m}$ from the presence of Fe III emission lines. At the same time, its radial velocity $\left(v_{\text {rad }}=26 \pm 3 \mathrm{~km} \mathrm{~s}^{-1}\right.$, Winkler \& Wolf 1989) suggests the distance $D=2.1 \mathrm{kpc}$, based on the galactic rotation curve, and leads to $\mathrm{L} \sim 10^{4} \mathrm{~L}_{\odot}$, which is comparable with those of other low-luminosity sample objects. Corporon \& Lagrange (1999) found radial velocity variations of $\mathrm{He} I$ lines which can be due to the presence of a secondary component. Analysing the ISO spectrum, Voors(1999) suggested that MWC 300 is surrounded by a long-lived dusty disk, which may be due to its binarity. 


\section{Conclusions}

We collected and analysed observational data for a group of galactic earlytype stars with unusual IR properties. Interstellar contribution to the overall reddening was estimated using the strengths of the diffuse interstellar band at $5780 \AA$. All the sample objects are likely to be surrounded by non-spherical gaseous and dusty envelopes. Their geometry, compact sizes, and an enhanced mass loss are most likely due to binarity of the objects.

Acknowledgments. We thank $\mathrm{O}$. Stahl for providing us with the spectra of MWC 300 and CPD $-52^{\circ} 9243$; G. Israelian for the spectra of HD 45677; T. Lloyd Evans, D. Kilkenny, F. Marang, and F. Van Wyk for obtaining the multicolor photometry at SAAO; K.S. Kuratov for making the 1-meter telescope of the Tien-Shan Observatory available to us; and R.H.M. Voors for providing us with a copy of his Ph.D. thesis. The spectra of HD 50138 from the Ritter Observatory of the University of Toledo (USA) were used in this study.

\section{References}

Bergner, Yu. K., et al. 1988, Izv. Glavn. Astron. Obs. v Pulkove 205, 142

Bergner, Yu. K., et al. 1990, Astrofizika 32, 203

Corporon, P., Lagrange, A.-M. 1999, A\&AS 136, 429

de Freitas Pacheco, J.A. 1998, In "B[e] stars", ed. C. Jaschek \& A.-M. Hubert, 221

Frontera, F., et al. 1998, A\&A 339, L69

Herbig, G.H. 1993, ApJ 407, 142

Langer, N., Heger, A. 1998, In "B[e] stars", ed. C. Jaschek \& A.-M. Hubert, 235

Lopes, D.F., Neto, A.D., de Freitas Pacheko, J.A. 1992, A\&A 262, 481

Lynden-Bell, D., Pringle, J.E. 1974, MNRAS 168, 603

Miroshnichenko, A.S., 1991, In "Infrared spectral region of stars", ed. C. Jaschek C. \& Y. Andrillat Y., 163

Miroshnichenko, A.S., et al. 1997, IBVS N4506

Schaerer, D., et al. 1993, A\&A 102, 339

Telting, J.H., et al. 1998, MNRAS 296, 785

Voors, R.H.M. 1999, Ph.D. Thesis, University of Utrecht

Winkler, H., Wolf, B. 1989, A\&A 219, 151

de Winter, D., van den Ancker, M.E. 1997, A\&AS 121, 275

Wolf, B., Stahl, O. 1985, A\&A 148, 412

Zickgraf, F.-J., Stahl, O. 1989, A\&A 223, 165

Zorec, J. 1998, In "B[e] stars", ed. C. Jaschek \& A.-M. Hubert A.-M., 27 